Portland State University

PDXScholar

\title{
Some New Results on Stochastic Comparisons of Coherent Systems Using Signatures
}

\author{
Ebrahim Amini-Seresht \\ Bu-Ali Sina University \\ Baha-Eldin Khaledi \\ Florida International University \\ Subhash C. Kochar \\ Portland State University, kochar@pdx.edu
}

Follow this and additional works at: https://pdxscholar.library.pdx.edu/mth_fac

Part of the Probability Commons

Let us know how access to this document benefits you.

Citation Details

Amini-Seresht, E., Khaledi, B. E., \& Kochar, S. (2020). Some new results on stochastic comparisons of coherent systems using signatures. Journal of Applied Probability, 57(1), 156-173.

This Post-Print is brought to you for free and open access. It has been accepted for inclusion in Mathematics and Statistics Faculty Publications and Presentations by an authorized administrator of PDXScholar. Please contact us if we can make this document more accessible: pdxscholar@pdx.edu. 


\title{
Some New Results on Stochastic Comparisons of Coherent Systems using Signatures
}

\author{
Ebrahim Amini-Seresht \\ Department of Statistics \\ Bu-Ali Sina University, Hamedan, Iran \\ E-mail: e.amini64@yahoo.com \\ Baha-Eldin Khaledi \\ Department of Statistics \\ Razi University, Kermanshah, Iran \\ E-mail: bkhaledi@hotmail.com \\ Subhash Kochar \\ Fariborz Maseeh Department of Mathematics and Statistics \\ Portland State University, Portrland, USA \\ E-mail:kochar@pdx.edu
}

June 21, 2019

\begin{abstract}
We consider coherent systems with independent and identically distributed components. While it is clear that the system's life will be stochastically larger when the components are replaced with stochastically better components, we show that, in general, similar results may not hold for hazard rate, reverse hazard rate and likelihood ratio orderings. We find sufficient conditions on the signature vector for these results to hold. These results are combined with the other well known results in the literature to get more general results for comparing two systems of the same size with different signature vectors and possibly with different independent and identically distributed component lifetimes. Some numerical examples are also provided to illustrate the theoretical results.
\end{abstract}

Key words: Likelihood ratio order; Hazard rate order; Reversed hazard rate order; Coherent system. 


\section{Introduction}

Consider a coherent system consisting of $n$ components with lifetimes $X_{1}, \ldots, X_{n}$, which are assumed to be independent and identically distributed (i.i.d.) continuous random variables. It follows from the coherent property of the system that the lifetime of the system $T_{\mathbf{X}}$ corresponds to exactly one of the order statistics, $X_{i: n}, i=1, \ldots, n$. [Samaniego (1985)] introduced the concept of "signature" of a system which depends on the design of the system. Let

$$
p_{i}=P\left[T_{\mathbf{X}}=X_{i: n}\right], i=1, \ldots, n \text { and } \sum_{i=1}^{n} p_{i}=1,
$$

be the probability that the system fails upon the occurrence of the $i t h$ component failure. The vector $\mathbf{p}=\left(p_{1}, \ldots, p_{n}\right)$ is called the signature of the system. The survival function of the lifetime of the underlying coherent system can be expressed as

$$
P\left(T_{\mathbf{X}}(\mathbf{p})>t\right)=\sum_{i=1}^{n} p_{i} P\left(X_{i: n}>t\right) .
$$

Because of the fundamental property of a systems signature p, namely, that the distribution of the system lifetime T, given i.i.d. components lifetimes with c.d.f. F, can be expressed as a function of $\mathrm{p}$ and $\mathrm{F}$ alone, we use the notation $T_{\mathbf{X}}(\mathbf{p}$ to denote the life time of the system.

This representation was used by to stochastically compare two systems with each with same i.i.d.components. These ordering properties are distribution-free in the sense that they do not depend on the common distribution of the components. As mentioned in Kochar et al. (1999) and also explained in Navarro et al. (2008), the above results continue to hold when the vector $\left(X_{1}, \ldots, X_{n}\right)$ has an exchangeable distribution. Moreover, they obtain distribution-free ordering properties used to compare systems having different numbers of exchangeable components. For further references on this topic, see Samaniego (2007) and Navarro et al. (2010)

First we review some notions of stochastic orderings. Throughout this paper increasing and decreasing stand for non-decreasing and non-increasing, respectively.

Let $X$ and $Y$ be two non-negative continuous random variables with density functions $f$ and $g$, distribution functions $F$ and $G$, survival functions $\bar{F}=1-F$ and $\bar{G}$, hazard rate functions $h_{X}=f / \bar{F}$ and $h_{Y}$, and reversed hazard rate functions $\tilde{h}_{X}=f / F$ and $\tilde{h}_{Y}$, respectively.

(a) $X$ is said to be larger than $Y$ in the likelihood ratio order (denoted by $X \geq_{l r} Y$ ) if $\frac{f(t)}{g(t)}$ is increasing in $t$.

(b) $X$ is said to be larger than $Y$ in the hazard rate order (denoted by $X \geq_{h r} Y$ ) if $\frac{\bar{F}(t)}{\bar{G}(t)}$ is increasing in $t$, or, equivalently, $h_{X}(t) \leq h_{Y}(t)$ for all $t$.

(c) $X$ is said to be larger than $Y$ in the reversed hazard rate order (denoted by $X \geq_{r h} Y$ ) if $\frac{F(t)}{G(t)}$ is increasing in $t$, or, equivalently, $\tilde{h}_{X}(t) \geq \tilde{h}_{Y}(t)$ for all $t$. 
(d) $X$ is said to be larger than $Y$ in the usual stochastic order (denoted by $X \geq_{s t} Y$ ) if $\bar{F}(t) \geq \bar{G}(t)$ for all $t$.

The above stochastic orders can be defined on the same lines to compare two discrete random variables with sample space $\{1, \ldots, n\}$. For an n-dimensional probability vector $\mathbf{p}=\left(p_{1}, \ldots, p_{n}\right)$, we denote by $h_{\mathbf{p}}(j)=\frac{p_{j}}{\sum_{i=j}^{n} p_{i}}$, the hazard rate of $\mathbf{p}$ and $\tilde{h}_{(\mathbf{j})}=\frac{p_{j}}{\sum_{i=1}^{j} p_{i}}$, as the reverse hazard rate of $\mathbf{p}$. For two discrete distributions $\mathbf{p}$ and $\mathbf{q}$ on the integers $\{1, \ldots, n\}$, we write

(a) $\mathbf{p} \geq_{s t} \mathbf{q}$ if and only if $\sum_{i=j}^{n} p_{i} \geq \sum_{i=j}^{n} q_{i}$ for $j=1, \ldots, n-1$.

(b) $\mathbf{p} \geq_{h r} \mathbf{q}$ if and only if $h_{\mathbf{p}}(i) \leq h_{\mathbf{q}}(i)$ for $i=1, \ldots, n$.

(c) $\mathbf{p} \geq_{r h r} \mathbf{q}$ if and only if $\tilde{h}_{\mathbf{p}}(i) \geq \tilde{h}_{\mathbf{q}}(i)$ for $i=1, \ldots, n$.

(d) $\mathbf{p} \geq \operatorname{lr} \mathbf{q}$ if and only if $\frac{p_{i}}{q_{i}}$ is increasing in $i$ for $i=1, \ldots, n$.

It is well known that

$$
X \geq_{l r} Y \Longrightarrow X \geq_{h r[r h]} Y \Longrightarrow X \geq_{s t} Y,
$$

but neither the reversed hazard rate nor hazard rate orders imply each other. one may refer to Shaked (2007) and Muller and Stoyan (2002) for more details.

Let $T_{\mathbf{Y}}(\mathbf{q})$ be the lifetime of another coherent system with signature vector $\mathbf{q}=\left(q_{1}, \ldots, q_{n}\right)$ and with component lifetimes $Y_{1}, \ldots, Y_{n}$. It is of interest to compare two systems $T_{\mathbf{X}}(\mathbf{p})$ and $T_{\mathbf{Y}}(\mathbf{q})$ according to various stochastic orders. Kochar et al. (1999) proved that if random lifetimes $X_{1}, \ldots, X_{n}$ are i.i.d., then

$$
\mathbf{p} \geq_{*} \mathbf{q} \Longrightarrow T_{\mathbf{X}}(\mathbf{p}) \geq_{*} T_{\mathbf{X}}(\mathbf{q})
$$

where $*$ order stands for $l r, h r, r h$ and $s t$ orders. In fact, they pointed out that the above results hold when $X_{1}, \ldots, X_{n}$ are exchangeable random variables and the corresponding consecutive order statistics are ordered according to $*$ ordering, that is if $X_{i+1: n} \geq_{*} X_{i: n} i=1,2, \ldots, n-$ 1. This problem has been pursued further by many other researchers. For instance, the reader may refer to [Belzunce et al.(2001a)], [Khaledi and Shaked (2007)], [Nanda et al. (1998)], [Navarro et al. (2008)], [Zhang (2010)] and [Zhang and Meeker (2013)], among others.

A $k$-out-of- $n$ system consisting of $n$ components with lifetimes $X_{1}, \ldots, X_{n}$, functions if and only if at least $k$ out of the $n$ components function. That is, the lifetime of the system corresponds to the $(n-k+1)$ th order statistic, $X_{(n-k+1: n)}$. Therefore, stochastically comparing two $k$-outof- $n$ systems is equivalent to comparing the corresponding order statistics. Let $X_{1}, \ldots, X_{n}$ and $Y_{1}, \ldots, Y_{n}$ be two sets of i.i.d. random variables. Then, it is known that

$$
X_{1} \geq_{*} Y_{1} \Longrightarrow X_{i: n} \geq_{*} Y_{i: n},
$$

where $*$ order stands for $l r, h r, r h$ and $s t$ orders. Note that $X_{i: n}$ and $Y_{i: n}$ are the lifetimes of two coherent systems with common signature vector $\mathbf{p}=\mathbf{q}=(0,0, \ldots, 0,1,0, \ldots, 0)$ ( 1 being 
at the $i t h$ position), but with different lifetime distributions. It is of interest to generalize the results of (1.4) to compare coherent systems with more general signature vectors.

It follows from the fact that

$$
X_{1} \geq_{s t} Y_{1} \Longrightarrow X_{i: n} \geq_{s t} Y_{i: n}, \text { for } i=1, \ldots, n
$$

and the equation (1.1) that when $X_{1}, \ldots, X_{n}\left(Y_{1}, \ldots, Y_{n}\right)$ are independent and identically distributed, then

$$
X_{1} \geq_{s t} Y_{1} \Rightarrow T_{\mathbf{X}}(\mathbf{p}) \geq_{s t} T_{\mathbf{Y}}(\mathbf{p})
$$

However, such a result may not hold for other stochastic orders like likelihood ratio, hazard rate and reverse hazard rate orders as shown in the following counter example.

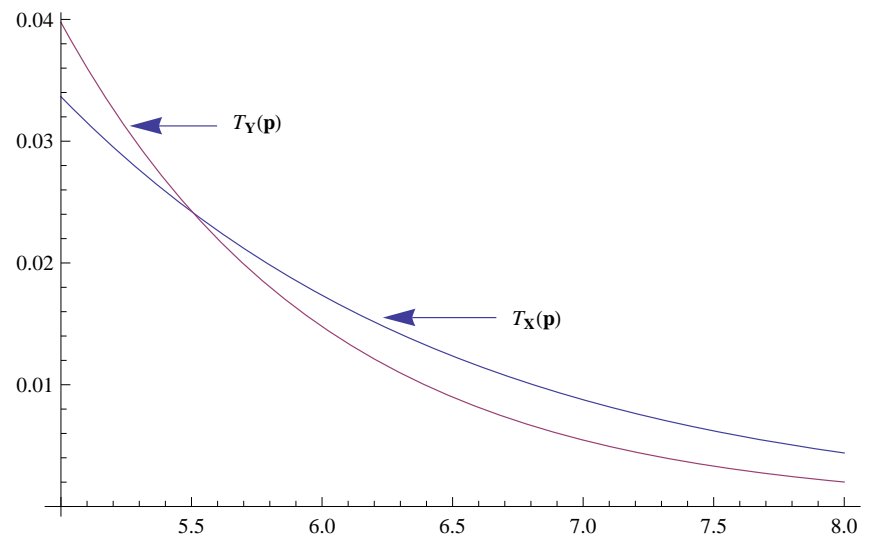

Figure 1: Plot of the hazard rate functions.

Example 1.1. Consider a coherent system of order 4 with lifetime

$$
T_{\mathbf{X}}(\mathbf{p})=\max \left(X_{1}, \min \left(X_{2}, X_{3}, X_{4}\right)\right)
$$

with signature vector $\mathbf{p}=\left(0, \frac{1}{2}, \frac{1}{4}, \frac{1}{4}\right)$, where $X_{i}, i=1, \ldots, 4$ are independent exponential random variables with common hazard rate 0.7. Let $Y_{i}, i=1, \ldots, 4$ be another set of independent exponential random variables with common hazard rate 1 . In Figure 1 we plot the hazard rate functions of $T_{X}(\mathbf{p})$ and $T_{Y}(\mathbf{p})$ and it can be seen that the hazard rate functions cross at $t=5.5$ even though $X_{1} \geq_{h r} Y_{1}$. Therefore, a result similar to (1.6) does not hold, in general, for hazard rate ordering.

In the second section, we find sufficient conditions on the signature vector $\mathbf{p}$ under which

$$
X_{1} \geq_{*} Y_{1} \Rightarrow T_{\mathbf{X}}(\mathbf{p}) \geq_{*} T_{\mathbf{Y}}(\mathbf{p})
$$

holds for hazard rate, reverse hazard and likelihood ratio orderings. This problem has also been studied by Navarro et al. (2013), but as will be seen later in the paper, their results seem to be 
different from ours. The rest of the paper is organized as follows. The main results of the paper are given in the next section. These new results are combined with the other well known results in the literature to get more general results when two systems of the same size with different signature vectors have differently distributed sets of component lifetimes. In Section 3, we will illustrate our results with some numerical examples and list all coherent systems of size 3,4 and 5 in Table 1 and 2 for which the conditions of Theorem 2.3 are satisfied. Results of the paper are summarized in the last Section.

\section{Main Results}

From (1.1) the survival function of $T_{\mathbf{X}}(\mathbf{p})$ can be written as

$$
\begin{aligned}
\bar{F}_{T_{\mathbf{X}}(\mathbf{p})}(t) & =\sum_{i=1}^{n} p_{i} \sum_{j=0}^{i-1}\left(\begin{array}{l}
n \\
j
\end{array}\right) F^{j}(t) \bar{F}^{n-j}(t) \\
& =\sum_{j=0}^{n-1}\left(\sum_{i=j+1}^{n} p_{i}\right)\left(\begin{array}{l}
n \\
j
\end{array}\right) F^{j}(t) \bar{F}^{n-j}(t),
\end{aligned}
$$

by changing the order of summation.

From this we find that the density function of $T_{\mathbf{X}}(\mathbf{p})$ as

$$
f_{T_{\mathbf{X}}(\mathbf{p})}(t)=\sum_{i=1}^{n} i p_{i}\left(\begin{array}{c}
n \\
i
\end{array}\right) F^{i-1}(t) \bar{F}^{n-i}(t) f(t) .
$$

and the hazard rate function of $T_{X}(\mathbf{p})$ is

$$
\begin{aligned}
h_{T_{X}(\mathbf{p})}(t) & =\frac{\sum_{i=1}^{n} i p_{i}\left(\begin{array}{c}
n \\
i
\end{array}\right) F^{i-1}(t) \bar{F}^{n-i+1}(t)}{\sum_{i=1}^{n} p_{i} \sum_{j=1}^{i-1}\left(\begin{array}{c}
n \\
j
\end{array}\right) F^{j}(t) \bar{F}^{n-j}(t)} h_{X}(t) \\
& =\frac{\sum_{i=0}^{n-1}(n-i) p_{i+1}\left(\begin{array}{c}
n \\
i
\end{array}\right) F^{i}(t) \bar{F}^{n-i}(t)}{\sum_{i=0}^{n-1}\left(\sum_{j=i+1}^{n} p_{j}\right)\left(\begin{array}{c}
n \\
i
\end{array}\right) F^{i}(t) \bar{F}^{n-i}(t)} h_{X}(t) \\
& =\Psi_{1}\left(\frac{F(t)}{\bar{F}(t)}\right) h_{X}(t)
\end{aligned}
$$

where

$$
\Psi_{1}(x)=\frac{\sum_{i=0}^{n-1}(n-i)\left(\begin{array}{c}
n \\
i
\end{array}\right) p_{i+1} x^{i}}{\sum_{i=0}^{n-1}\left(\sum_{j=i+1}^{n} p_{j}\right)\left(\begin{array}{c}
n \\
i
\end{array}\right) x^{i}}
$$

The reverse hazard rate function of $T_{X}(\mathbf{p})$ is

$$
\begin{aligned}
\tilde{h}_{T_{\mathbf{X}}(\mathbf{p})}(t) & =\frac{\sum_{i=1}^{n} i p_{i}\left(\begin{array}{c}
n \\
i
\end{array}\right) F^{i}(t) \bar{F}^{n-i}(t)}{\sum_{i=1}^{n}\left(\sum_{j=1}^{i} p_{j}\right)\left(\begin{array}{c}
n \\
i
\end{array}\right) F^{i}(t) \bar{F}^{n-i}(t)} \tilde{h}_{X}(t) \\
& =\Psi_{2}(x)\left(\frac{F(t)}{\bar{F}(t)}\right) \tilde{h}_{X}(t),
\end{aligned}
$$


where

$$
\Psi_{2}(x)=\frac{\sum_{i=1}^{n} i p_{i}\left(\begin{array}{c}
n \\
i
\end{array}\right) x^{1}}{\sum_{i=1}^{n}\left(\sum_{j=1}^{i} p_{j}\right)\left(\begin{array}{c}
n \\
i
\end{array}\right) x^{i}(t)}
$$

First we prove the following lemma which gives simple sufficient conditions for the functions $\Psi_{1}$ and $\Psi_{2}$ to be monotone.

Lemma 2.1. For $i=n_{1} \ldots, n_{2}$, let $c_{i}$ and $d_{i}$ be nonnegative constants. If $\ell_{i}=\frac{c_{i}}{d_{i}}$ is increasing (decreasing) in $i$, then so is

$$
\phi(x)=\frac{\sum_{i=n_{i}}^{n_{2}} c_{i} x^{i}}{\sum_{i=n_{1}}^{n_{2}} d_{i} x^{i}}
$$

on the set of positive numbers $x$.

Proof. We only give the proof when $\ell_{i}$ is increasing in $i$.

Differentiating $\phi(x)$ with respect to $x$, we obtain a fraction with positive denominator and numerator equal to

$$
\begin{aligned}
\phi^{\prime}(x) & \stackrel{\operatorname{sgn}}{=}\left(\sum_{i=n_{1}}^{n_{2}} i c_{i} x^{i-1}\right)\left(\sum_{j=n_{1}}^{n_{2}} d_{j} x^{j}\right)-\left(\sum_{i=n_{1}}^{n_{2}} i d_{i} x^{i-1}\right)\left(\sum_{j=n_{1}}^{n_{2}} c_{j} x^{j}\right) \\
& =\sum_{i=n_{1}}^{n_{2}} \sum_{j=n_{1}}^{n_{2}}\left[i c_{i} d_{j}-i d_{i} c_{j}\right] x^{i-1} x^{j} \\
& =\sum_{k}\left[\sum_{(i, j) \in\left\{n_{1}, \ldots, n_{2}\right\}: i+j-1=k}\left(i c_{i} d_{j}-i d_{i} c_{j}\right)\right] x^{k}
\end{aligned}
$$

Decomposing the inner sum according to $i<j$ and $i>j$ and after renaming the indices, we get

$$
\sum_{k}\left[\sum_{(i, j) \in\left\{n_{1}, \ldots, n_{2}\right\}: i+j-1=k}(i-j)\left(i c_{i} d_{j}-i d_{i} c_{j}\right)\right] x^{k},
$$

If $\ell$ is increasing, then all the coefficients of $x^{k}$ are nonnegative, thus proving our lemma.

Using the above lemma, we prove the following result.

Lemma 2.2. Let $\mathbf{p}=\left(p_{1}, \ldots, p_{n}\right)$ be a probability vector.

(a) If $(n-i) h_{\mathbf{p}}(i+1)$ is increasing in $i$ for $i \in\{0, \ldots, n-1\}$, then

$$
\Psi_{1}(x)=\frac{\sum_{i=0}^{n-1}(n-i) p_{i+1}\left(\begin{array}{c}
n \\
i
\end{array}\right) x^{i}}{\sum_{i=0}^{n-1}\left(\begin{array}{c}
n \\
i
\end{array}\right) \sum_{j=i+1}^{n} p_{j} x^{i}},
$$

is increasing in $x \geq 0$. 
(b) If $i \tilde{h}_{\mathbf{p}}(i)$ is decreasing in $i$ for $i \in\{1, \ldots, n\}$, then

$$
\Psi_{2}(x)=\frac{\sum_{i=1}^{n} i\left(\begin{array}{c}
n \\
i
\end{array}\right) p_{i} x^{i}}{\sum_{i=1}^{n}\left(\begin{array}{c}
n \\
i
\end{array}\right) \sum_{j=1}^{i} p_{j} x^{i}}
$$

is decreasing in $x \geq 0$.

\section{Proof}

(a) Letting $c_{i}=(n-i)\left(\begin{array}{c}n \\ i\end{array}\right) p_{i+1}$ and $d_{i}=\left(\begin{array}{c}n \\ i\end{array}\right) \sum_{j=i+1}^{n} p_{j}$, we find that $\ell_{i}=\frac{c_{i}}{d_{i}}=(n-i) h_{\mathbf{p}}(i+1)$ is increasing in $i$ under the given condition. By taking $n_{1}=0, n_{2}=n-1$, it follows from Lemma 2.2 that $\Psi_{1}(x)$ is increasing in $x$.

(b) Letting $c_{i}=i\left(\begin{array}{c}n \\ i\end{array}\right) p_{i}$ and $d_{i}=\left(\begin{array}{c}n \\ i\end{array}\right) \sum_{j=1}^{i} p_{j}$, we find that $\ell_{i}=\frac{c_{i}}{d_{i}}=\ell_{i}=i \tilde{h}_{\mathbf{p}}(i)$ is decreasing in $i \in\{1, \ldots, n\}$ under the given condition. By taking $n_{1}=1, n_{2}=n$, it follows from Lemma 2.2 that $\Psi_{2}(x)$ is decreasing in $x$.

The rational function $\Psi_{1}(x)$ seems to appear at several places in the literature. For example, Samaniego (1985) proved that a coherent system with $n$ i.i.d. IFR (increasing failure rate) components is IFR if and only if the function $\Psi_{1}(x)$ is increasing in $x \in(0, \infty)$. A similar result holds for a coherent system with DFR components. The above lemma gives simple conditions in terms of the hazard rate and the reverse hazards rates of the signature vector for the monotonicity of the functions $\Psi_{1}$ and $\Psi_{2}$.

Now we state the main result of this paper.

Theorem 2.3. Let $T_{\mathbf{X}}(\mathbf{p})$ be the lifetime of a coherent system with signature vector $\mathbf{p}$ and with the components lifetimes $X_{1}, \ldots, X_{n}$ which are independent with a common continuous distribution $F$ and density function $f$. Let $Y_{1}, \ldots, Y_{n}$ be i.i.d. with common continuous distribution $G$ and density function $g$. Then

(a) if

$$
(n-j) h(j+1) \text { is increasing in } j \text { for any } j=0, \ldots, n-1 \text {, }
$$

then

$$
X_{1} \geq_{h r} Y_{1} \Longrightarrow T_{\mathbf{X}}(\mathbf{p}) \geq_{h r} T_{\mathbf{Y}}(\mathbf{p})
$$

(b) If

$$
j \tilde{h}(j) \text { is decreasing in } j \text { for any } j=1, \ldots, n \text {, }
$$

then

$$
X_{1} \geq_{r h} Y_{1} \Longrightarrow T_{\mathbf{X}}(\mathbf{p}) \geq_{r h} T_{\mathbf{Y}}(\mathbf{p}),
$$

(c) Let $\Phi(u)=\sum_{j=0}^{n} \xi(j) u^{n-j}$, for all $u \in(0,1)$ where $\xi(j)=\sum_{i=j}^{n} p_{i+1}(-1)^{i-j}\left(\begin{array}{l}n-j-1 \\ n-i-1\end{array}\right)\left(\begin{array}{l}n \\ j\end{array}\right)(n-$ $j), j=0, \ldots, n$. If

$$
\frac{h_{X}(t)}{h_{Y}(t)} \text { is increasing in } t
$$

and for some point $v \in(0,1)$, 
(i) $\frac{u \Phi^{\prime}(u)}{\Phi(u)}$ is decreasing and positive for all $u \in(0, v)$,

(ii) $\frac{(1-u) \Phi^{\prime}(u)}{\Phi(u)}$ is decreasing and negative for all $u \in(v, 1)$.

Then,

$$
X_{1} \geq_{l r} Y_{1} \Longrightarrow T_{\mathbf{X}}(\mathbf{p}) \geq_{l r} T_{\mathbf{Y}}(\mathbf{p})
$$

\section{Proof}

(a) From (2.3),

$$
\begin{aligned}
h_{T_{X}(\mathbf{p})}(t) & =\Psi_{1}\left(\frac{F(t)}{\bar{F}(t)}\right) h_{X}(t) \\
& \leq \Psi_{1}\left(\frac{G(t)}{\bar{G}(t)}\right) h_{Y}(t) \\
& =h_{T_{X}(\mathbf{p})}(t),
\end{aligned}
$$

as $\Psi_{1}(x)$ is increasing in $x>0$ and $h_{X}(t) \leq h_{Y}(t)$ for all $t$ and the fact that $h_{X}(t) \leq$ $h_{Y}(t) \Rightarrow F(t) \leq G(t)$ for all $t$.

(b) The proof is similar to that of part (a) above and hence omitted.

(c) It is known that the survival function of $X_{i: n}$ can be written as (see [Gupta $(2002$, p. 839)])

$$
P\left(X_{i: n}>t\right)=\sum_{j=0}^{i-1}(-1)^{i-j-1}\left(\begin{array}{c}
n \\
j
\end{array}\right)\left(\begin{array}{c}
n-j-1 \\
n-i
\end{array}\right) \bar{F}^{n-j}(t),
$$

from which its density function is

$$
f_{i: n}(t)=h_{X}(t) \sum_{j=0}^{i-1}(-1)^{i-j-1}\left(\begin{array}{c}
n \\
j
\end{array}\right)\left(\begin{array}{c}
n-j-1 \\
n-i
\end{array}\right)(n-j) \bar{F}^{n-j}(t),
$$

where $h_{X}(t)$ is the hazard rate function of $X$. Hence, it follows that

$$
\begin{aligned}
f_{T_{\mathbf{X}}(\mathbf{p})}(t) & =h_{X}(t) \sum_{i=1}^{n} \sum_{j=0}^{i-1} p_{i}(-1)^{i-j-1}\left(\begin{array}{c}
n \\
j
\end{array}\right)\left(\begin{array}{c}
n-j-1 \\
n-i
\end{array}\right)(n-j) \bar{F}^{n-j}(t) \\
& =h_{X}(t) \sum_{j=0}^{n} \sum_{i=j}^{n} p_{i+1}(-1)^{i-j}\left(\begin{array}{c}
n-j-1 \\
n-i-1
\end{array}\right)\left(\begin{array}{c}
n \\
j
\end{array}\right)(n-j) \bar{F}^{n-j}(t) \\
& =h_{X}(t) \sum_{j=0}^{n} \xi(j) \bar{F}^{n-j}(t)
\end{aligned}
$$

where $\xi(j)=\sum_{i=j}^{n} p_{i+1}(-1)^{i-j}\left(\begin{array}{c}n-j-1 \\ n-i-1\end{array}\right)\left(\begin{array}{c}n \\ j\end{array}\right)(n-j), j=0, \ldots, n$.

The required result is equivalent to proving that

$$
\frac{f_{T_{\mathbf{X}}(\mathbf{p})}(t)}{f_{T_{\mathbf{Y}}(\mathbf{p})}(t)}=\frac{h_{X}(t)}{h_{Y}(t)} \cdot \frac{\sum_{j=0}^{n} \xi(j) \bar{F}^{n-j}(t)}{\sum_{j=0}^{n} \xi(j) \bar{G}^{n-j}(t)},
$$


is increasing in $t$. From the assumption that $\frac{h_{X}(t)}{h_{Y}(t)}$ is increasing in $t$, we only need to show that

$$
\Delta(t)=\frac{\Phi(\bar{F}(t))}{\Phi(\bar{G}(t))}
$$

is increasing in $t$, where, $\Psi(u)=\sum_{j=0}^{n} \xi(j) u^{n-j}(t)$.

Suppose that for some $x_{v}, \bar{F}\left(x_{v}\right)=v$.

Case 1: For all $t \in\left(x_{v}, \infty\right)$, i.e., $\bar{F}(t) \in(0, v)$, the sign of the derivative $\Delta(t)$ is

$$
\begin{aligned}
\Delta^{\prime}(t) & ={ }^{s g n} \quad g(t) \frac{\Phi^{\prime}(\bar{G}(t))}{\Phi(\bar{G}(t))}-f(t) \frac{\Phi^{\prime}(\bar{F}(t))}{\Phi(\bar{F}(t))} \\
& =h_{Y}(t) \frac{\bar{G}(t) \Phi^{\prime}(\bar{G}(t))}{\Phi(\bar{G}(t))}-h_{X}(t) \frac{\bar{F}(t) \Phi^{\prime}(\bar{F}(t))}{\Phi(\bar{F}(t))} \\
& \geq h_{Y}(t)\left[\frac{\bar{G}(t) \Phi^{\prime}(\bar{G}(t))}{\Phi(\bar{G}(t))}-\frac{\bar{F}(t) \Phi^{\prime}(\bar{F}(t))}{\Phi(\bar{F}(t))}\right] \\
& =h_{Y}(t)\left[\frac{u_{1} \Phi^{\prime}\left(u_{1}\right)}{\Phi\left(u_{1}\right)}-\frac{u_{2} \Phi^{\prime}\left(u_{2}\right)}{\Phi\left(u_{2}\right)}\right] \\
& \geq 0
\end{aligned}
$$

where $\bar{G}(t)=u_{1}$ and $\bar{F}(t)=u_{2}$. The first inequality follows from the assumptions that $\frac{u \Psi^{\prime}(u)}{\Psi(u)}$ is positive and the fact that $X \geq_{l r} Y \Longrightarrow X \geq_{h r} Y$. It follows from $X \geq_{l r} Y \Longrightarrow$ $X \geq_{s t} Y$ that $u_{1} \leq u_{2}$. Now using this observation, the second inequality follows from the assumption that $\frac{u \Psi^{\prime}(u)}{\Psi(u)}$ is decreasing and positive for $u \in(0, v)$.

Case 2: For all $t \in\left(0, x_{v}\right)$, i.e., $\bar{F}(t) \in(v, 1)$, the sign of the derivative $\Delta(t)$ with respect to $t$ is

$$
\begin{aligned}
\Delta^{\prime}(t) & ={ }^{s g n} \quad g(t) \frac{\Phi^{\prime}(\bar{G}(t))}{\Phi(\bar{G}(t))}-f(t) \frac{\Phi^{\prime}(\bar{F}(t))}{\Phi(\bar{F}(t))} \\
& =\tilde{h}_{Y}(t) \frac{(1-\bar{G}(t)) \Phi^{\prime}(\bar{G}(t))}{\Phi(\bar{G}(t))}-\tilde{h}_{X}(t) \frac{(1-\bar{F}(t)) \Phi^{\prime}(\bar{F}(t))}{\Phi(\bar{F}(t))} \\
& =\tilde{h}_{X}(t)\left[-\frac{(1-\bar{F}(t)) \Phi^{\prime}(\bar{F}(t))}{\Phi(\bar{F}(t))}\right]-\tilde{h}_{Y}(t)\left[-\frac{(1-\bar{G}(t)) \Phi^{\prime}(\bar{G}(t))}{\Phi(\bar{G}(t))}\right] \\
& \geq \tilde{h}_{Y}(t)\left[-\frac{\left(1-u_{2}\right) \Phi^{\prime}\left(u_{2}\right)}{\Phi\left(u_{2}\right)}\right]-\tilde{h}_{Y}(t)\left[-\frac{\left(1-u_{1}\right) \Phi^{\prime}\left(u_{1}\right)}{\Phi\left(u_{1}\right)}\right] \\
& \geq 0,
\end{aligned}
$$

where $\bar{G}(t)=u_{1}$ and $\bar{F}(t)=u_{2}$. The first inequality follows from the assumptions that $-\frac{(1-u) \Phi^{\prime}(u)}{\Phi(u)}$ is positive for all $u \in(v, 1)$ and the fact that $X \geq_{l r} Y \Longrightarrow X \geq_{r h} Y$. It follows from $X \geq_{l r} Y \Longrightarrow X \geq_{s t} Y$ that $u_{1} \leq u_{2}$. Now using this observation, the second inequality follows from the assumption that $-\frac{(1-u) \Phi^{\prime}(u)}{\Phi(u)}$ is increasing and positive in $u \in(v, 1)$. 
Equations (2.8) and (2.10) give simple sufficient conditions on the signature vector for two systems to be ordered according to hazard rate and reverse hazard rate orderings. This problem has also been studied by Navarro et al. (2013). However, as seen by examples in the next section, their results are different from results of this paper. Kochar et al. (1999) established the following results for comparing two coherent systems with signature vectors $\mathbf{p}$ and $\mathbf{q}$, but with i.i.d.component lifetimes $X_{1}, \ldots X_{n}$.

Theorem 2.4. (Kochar et al.1999) Let $\mathbf{p}$ and $\mathbf{q}$ be the signature vectors of two coherent systems with same number of components and let $T_{\mathbf{X}}(\mathbf{p})$ and $T_{\mathbf{X}}(\mathbf{q})$ be their lifetimes, where the the elements of the vector $\mathbf{X}$ are i.i.d. Then

(a) $\mathbf{p} \geq_{s t} \mathbf{q} \Rightarrow T_{\mathbf{X}}(\mathbf{p}) \geq_{s t} T_{\mathbf{X}}(\mathbf{p})$

(b) $\mathbf{p} \geq_{h r} \mathbf{q} \Rightarrow T_{\mathbf{X}}(\mathbf{p}) \geq_{h r} T_{\mathbf{X}}(\mathbf{p})$

(c) $\mathbf{p} \geq_{l r} \mathbf{q} \Rightarrow T_{\mathbf{X}}(\mathbf{p}) \geq_{l r} T_{\mathbf{X}}(\mathbf{p})$

A similar result holds for reverse hazard rate ordering. Combining the results of the above theorem with the previous theorem, we obtain the following general results which show how the hazard rate, the reversed hazard rate and the likelihood ratio orders between $X$ and $Y$ and signature vectors $\mathbf{p}$ and $\mathbf{q}$ are preserved by the lifetimes of the corresponding coherent systems.

Theorem 2.5. Under the assumptions of Theorem 2.3

(a) if either $(n-j) h_{\mathbf{p}}(j)$ or $(n-j) h_{\mathbf{q}}(j)$ is increasing in $j \in\{j=0, \ldots, n-1\}$, then $\mathbf{p} \geq_{h r} \mathbf{q} \Rightarrow T_{\mathbf{X}}(\mathbf{p}) \geq_{h r} T_{\mathbf{Y}}(\mathbf{q})$.

(b) if either $j \tilde{h_{\mathbf{p}}}(j)$ or $j \tilde{h_{\mathbf{q}}}(j)$ is decreasing in $j$ for $j=1, \ldots$, $n$, then $\mathbf{p} \geq_{r h} \mathbf{q} \Rightarrow T_{\mathbf{X}}(\mathbf{p}) \geq_{r h}$ $T_{\mathbf{Y}}(\mathbf{q})$.

(c) if the conditions of Theorem 2.3 (c) are satisfied by either $\mathbf{p}$ or $\mathbf{q}$, then $\mathbf{p} \geq_{l r} \mathbf{q} \Rightarrow$ $T_{\mathbf{X}}(\mathbf{p}) \geq_{l r} T_{\mathbf{Y}}(\mathbf{q})$.

\section{Some numerical examples}

In this section, we present some coherent systems that satisfy the conditions of Theorem 2.3.

Example 3.1. Consider a coherent system of order 4 with lifetime

$$
T_{\mathbf{X}}(\mathbf{p})=\min \left(X_{1}, \max \left(X_{2}, X_{3}, X_{4}\right)\right)
$$

It is easy to show that the signature vector of this system is $\mathbf{p}=\left(\frac{1}{4}, \frac{1}{4}, \frac{1}{2}, 0\right)$. Note that the condition of " $(n-j) h(j+1)$ is increasing in $j$ " is equivalent to that $\frac{(n-r) p_{r+1}}{(n-k) p_{k+1}} \geq \frac{\sum_{j=r+1}^{n} p_{j}}{\sum_{j=k+1}^{n} p_{j}}$ for all $r \geq k$, whenever $p_{k+1}>0$. Below we check this condition for various cases of $k$ and $r$. 
- If $r=3$ and $k=2$, then $\frac{(n-r) p_{r+1}}{(n-k) p_{k+1}}=0 \geq \frac{\sum_{j=r+1}^{n} p_{j}}{\sum_{j=k+1}^{n} p_{j}}=0$.

- If $r=3$ and $k=1$, then $\frac{(n-r) p_{r+1}}{(n-k) p_{k+1}}=0 \geq \frac{\sum_{j=r+1}^{n} p_{j}}{\sum_{j=k+1}^{n} p_{j}}=0$.

- If $r=2$ and $k=1$, then $\frac{(n-r) p_{r+1}}{(n-k) p_{k+1}}=\frac{8}{3} \geq \frac{\sum_{j=r+1}^{n} p_{j}}{\sum_{j=k+1}^{n} p_{j}}=\frac{4}{3}$.

- If $r=1$ and $k=0$, then $\frac{(n-r) p_{r+1}}{(n-k) p_{k+1}}=\frac{3}{4} \geq \frac{\sum_{j=r+1}^{n} p_{j}}{\sum_{j=k+1}^{n} p_{j}}=\frac{3}{4}$.

That is, this system satisfies condition (a) of Theorem 2.3.

Example 3.2. Consider a coherent system of order 4 with lifetime

$$
T_{\mathbf{X}}(\mathbf{p})=\max \left(X_{1}, \min \left(X_{2}, X_{3}, X_{4}\right)\right)
$$

It is easy to show that the signature vector of this system is $\mathbf{p}=\left(0, \frac{1}{2}, \frac{1}{4}, \frac{1}{4}\right)$. Note that the condition of " $j \tilde{h}(j)$ is decreasing in $j$ " is equivalent to that $\frac{\sum_{i=1}^{r} p_{i}}{\sum_{i=1}^{k} p_{i}} \geq \frac{r p_{r}}{k p_{k}}$ for all $r \geq k$, whenever $p_{k}>0$. The above inequality is evaluated for various possible values of $k$ and $r$.

- If $r=3$ and $k=2$, then $\frac{\sum_{i=1}^{r} p_{i}}{\sum_{i=1}^{k} p_{i}}=\frac{3}{2} \geq \frac{r p_{r}}{k p_{k}}=\frac{3}{4}$.

- If $r=4$ and $k=3$, then $\frac{\sum_{i=1}^{r} p_{i}}{\sum_{i=1}^{k} p_{i}}=\frac{4}{3} \geq \frac{r p_{r}}{k p_{k}}=\frac{4}{3}$.

- If $r=4$ and $k=2$, then $\frac{\sum_{i=1}^{r} p_{i}}{\sum_{i=1}^{k} p_{i}}=2 \geq \frac{r p_{r}}{k p_{k}}=1$.

That is, the condition in Theorem 2.3 (b) is satisfied by such a system.

Example 3.3. Consider a coherent system of order 4 with signature vector $\mathbf{p}=\left(0, \frac{1}{3}, \frac{2}{3}, 0\right)$. It can be seen that

$$
\begin{aligned}
\Psi(u) & =\xi(0) u^{4}+\xi(1) u^{3}+\xi(2) u^{2} \\
& =4 u^{4}-12 u^{3}+8 u^{2}
\end{aligned}
$$

It can be seen that

$$
\Delta_{1}(u)=\frac{u \Psi^{\prime}(u)}{\Psi(u)}=\frac{16 u^{4}-36 u^{3}+16 u^{2}}{4 u^{4}-12 u^{3}+8 u^{2}}
$$

and

$$
\Delta_{2}(u)=\frac{(1-u) \Psi^{\prime}(u)}{\Psi(u)}=\frac{(1-u)\left(16 u^{3}-36 u^{2}+16 u\right)}{4 u^{4}-12 u^{3}+8 u^{2}} .
$$

The graphs of the functions $\Delta_{1}(u)$ and $\Delta_{2}(u)$ are given in Figures 2 and 3, respectively. It is seen that $\Delta_{1}(u)$ is decreasing and is positive in $u \in(0,0.61)$ and $\Delta_{2}(u)$ is decreasing and is negative in $u \in(0.61,1)$, which is shows that the conditions (i) and (ii) of Theorem 2.3 (c) are satisfied. 


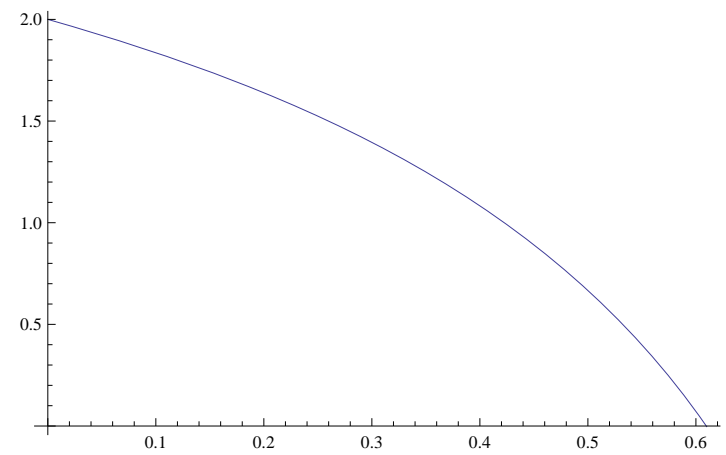

Figure 2: Plot of $\Delta_{1}(u)$, for all $u \in(0,0.61)$

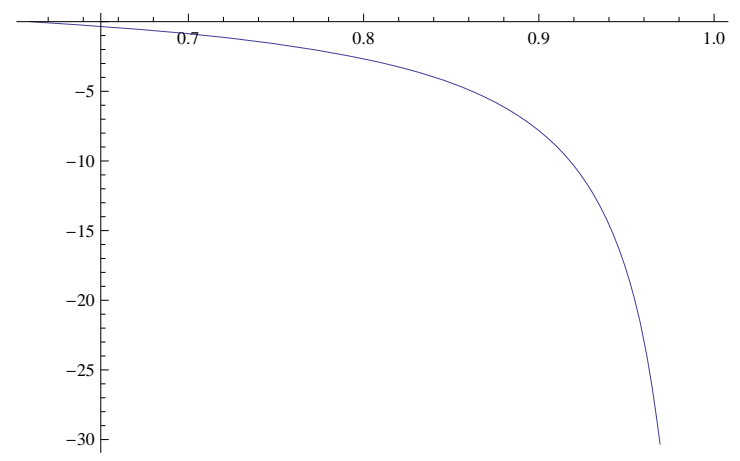

Figure 3: Plot of $\Delta_{2}(u)$, for all $u \in(0.61,1)$

Example 3.4. Consider a coherent system of order 4 with signature vector $\mathbf{p}=\left(0, \frac{1}{2}, \frac{1}{2}, 0\right)$. It can be seen that

$$
\begin{aligned}
\Psi(u) & =\xi(0) u^{4}+\xi(1) u^{3}+\xi(2) u^{2} \\
& =-6 u^{3}+6 u^{2} \\
\Delta_{3}(u) & =\frac{u \Psi^{\prime}(u)}{\Psi(u)}=\frac{12 u^{2}-18 u^{3}}{6 u^{2}-6 u^{3}}
\end{aligned}
$$

and

$$
\Delta_{4}(u)=\frac{(1-u) \Psi^{\prime}(u)}{\Psi(u)}=\frac{(1-u)\left(12 u-18 u^{2}\right)}{6 u^{2}-6 u^{3}} .
$$

The graphs of the functions $\Delta_{3}(u)$ and $\Delta_{4}(u)$, are given in Figures 4 and 5 , respectively. It is seen that $\Delta_{3}(u)$ is decreasing and is positive in $u \in(0,0.667)$ and $\Delta_{2}(u)$ is decreasing and is negative in $u \in(0.667,1)$, which is shows that conditions (i) and (ii) of Theorem 2.3 (c) are satisfied.

In Tables 1 and 2, we characterize all possible coherent systems of sizes 3, 4 and 5, for which the results of Theorem 2.3 (a) and (b) hold. 


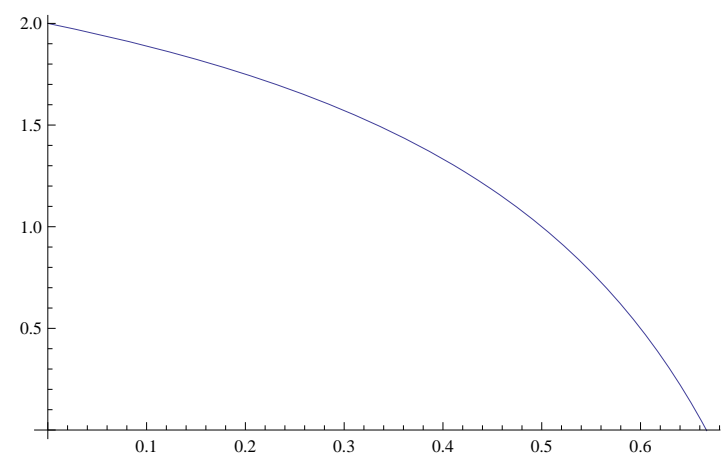

Figure 4: Plot of $\Delta_{3}(u)$, for all $u \in(0,0.667)$

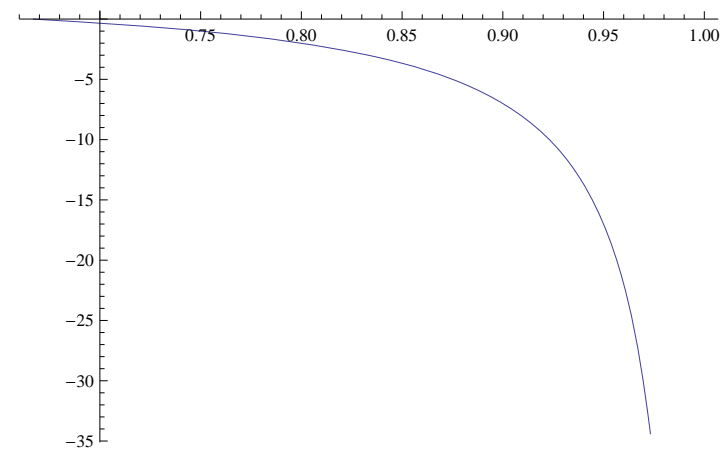

Figure 5: Plot of $\Delta_{4}(u)$, for all $u \in(0.667,1)$

Table 1. Coherent systems of sizes 3 and 4

\begin{tabular}{lcc}
\hline $\mathbf{p}$ & $(n-j) h(j+1)$ & $j \tilde{r}(j)$ \\
\hline$\left(\frac{1}{3}, \frac{2}{3}, 0\right)$ & $\uparrow j$ & \\
$\left(0, \frac{2}{3}, \frac{1}{3}\right)$ & & $\downarrow j$ \\
& & \\
\hline $\mathbf{p}$ & $(n-j) h(j+1)$ & $j \tilde{r}(j)$ \\
\hline$\left(\frac{1}{2}, \frac{1}{2}, 0,0\right)$ & $\uparrow j$ & \\
$\left(\frac{1}{4}, \frac{3}{4}, 0,0\right)$ & $\uparrow j$ & \\
$\left(\frac{1}{4}, \frac{1}{4}, \frac{1}{2}, 0\right)$ & $\uparrow j$ & \\
$\left(\frac{1}{4}, \frac{7}{12}, \frac{1}{6}, 0\right)$ & $\uparrow j$ & \\
$\left(0, \frac{5}{6}, \frac{1}{6}, 0\right)$ & $\uparrow j$ & $\downarrow j$ \\
$\left(0, \frac{1}{3}, \frac{1}{3}, 0\right)$ & $\uparrow j$ & $\downarrow j$ \\
$\left(0, \frac{2}{3}, \frac{1}{3}, 0\right)$ & $\uparrow j$ & $\downarrow j$ \\
$\left(0, \frac{1}{2}, \frac{1}{2}, 0\right)$ & $\uparrow j$ & $\downarrow j$ \\
$\left(0, \frac{1}{3}, \frac{2}{3}, 0\right)$ & $\uparrow j$ & $\downarrow j$ \\
$\left(0, \frac{1}{3}, \frac{2}{3}, 0\right)$ & $\uparrow j$ & $\downarrow j$ \\
$\left(0, \frac{1}{6}, \frac{5}{6}, 0\right)$ & $\uparrow j$ & $\downarrow j$ \\
$\left(0,0, \frac{1}{2}, \frac{1}{2}\right)$ & & $\downarrow j$ \\
$\left(0,0, \frac{3}{4}, \frac{1}{4}\right)$ & & $\downarrow j$ \\
$\left(0, \frac{1}{2}, \frac{1}{4}, \frac{1}{4}\right)$ & & $\downarrow j$ \\
$\left(0, \frac{1}{6}, \frac{7}{12}, \frac{1}{4}\right)$ & & $\downarrow j$ \\
\hline
\end{tabular}


Table 2. Coherent systems of size 5

\begin{tabular}{|c|c|c|}
\hline $\mathrm{p}$ & $(n-j) h(j+1)$ & $j \tilde{r}(j)$ \\
\hline$\left(\frac{3}{5}, \frac{2}{5}, 0,0,0\right)$ & $\uparrow j$ & \\
\hline$\left(\frac{2}{5}, \frac{3}{5}, 0,0,0\right)$ & $\uparrow j$ & \\
\hline$\left(\frac{1}{5}, \frac{4}{5}, 0,0,0\right)$ & $\uparrow j$ & \\
\hline$\left(\frac{2}{5}, \frac{1}{2}, \frac{1}{10}, 0,0\right)$ & $\uparrow j$ & \\
\hline$\left(\frac{1}{5}, \frac{7}{10}, \frac{1}{10}, 0,0\right)$ & $\uparrow j$ & \\
\hline$\left(\frac{2}{5}, \frac{3}{10}, \frac{3}{10}, 0,0\right)$ & $\uparrow j$ & \\
\hline$\left(\frac{1}{5}, \frac{3}{5}, \frac{1}{5}, 0,0\right)$ & $\uparrow j$ & \\
\hline$\left(\frac{1}{5}, \frac{1}{2}, \frac{3}{10}, 0,0\right)$ & $\uparrow j$ & \\
\hline$\left(\frac{1}{5}, \frac{2}{5}, \frac{2}{5}, 0,0\right)$ & $\uparrow j$ & \\
\hline$\left(\frac{1}{5}, \frac{3}{10}, \frac{1}{2}, 0,0\right)$ & $\uparrow j$ & \\
\hline$\left(\frac{1}{5}, \frac{1}{5}, \frac{3}{5}, 0,0\right)$ & $\uparrow j$ & \\
\hline$\left(\frac{1}{5}, \frac{1}{2}, \frac{1}{5}, \frac{1}{10}, 0\right)$ & $\uparrow j$ & \\
\hline$\left(\frac{1}{5}, \frac{3}{10}, \frac{2}{5}, \frac{1}{10}, 0\right)$ & $\uparrow j$ & \\
\hline$\left(\frac{1}{5}, \frac{1}{5}, \frac{1}{2}, \frac{1}{10}, 0\right)$ & $\uparrow j$ & \\
\hline$\left(\frac{1}{5}, \frac{1}{5}, \frac{2}{5}, \frac{1}{5}, 0\right)$ & $\uparrow j$ & \\
\hline$\left(\frac{1}{5}, \frac{1}{5}, \frac{1}{5}, \frac{2}{5}, 0\right)$ & $\uparrow j$ & \\
\hline$\left(0,0,0, \frac{3}{5}, \frac{2}{5}\right)$ & & $\downarrow j$ \\
\hline$\left(0,0,0 \frac{2}{5}, \frac{3}{5}\right)$ & & $\downarrow j$ \\
\hline$\left(0,0,0, \frac{1}{5}, \frac{4}{5}\right)$ & & $\downarrow j$ \\
\hline$\left(0,0, \frac{2}{5}, \frac{1}{2}, \frac{1}{10}\right)$ & & $\downarrow j$ \\
\hline$\left(0,0, \frac{1}{5}, \frac{7}{10}, \frac{1}{10}\right)$ & & $\downarrow j$ \\
\hline$\left(0,0, \frac{2}{5}, \frac{3}{10}, \frac{3}{10}\right)$ & & $\downarrow j$ \\
\hline$\left(0,0, \frac{1}{5}, \frac{3}{5}, \frac{1}{5}\right)$ & & $\downarrow j$ \\
\hline$\left(0,0, \frac{1}{5}, \frac{1}{2}, \frac{3}{10}\right)$ & & $\downarrow j$ \\
\hline$\left(0,0, \frac{1}{5}, \frac{2}{5}, \frac{2}{5}\right)$ & & $\downarrow j$ \\
\hline$\left(0,0, \frac{1}{5}, \frac{3}{10}, \frac{1}{2}\right)$ & & $\downarrow j$ \\
\hline$\left(0,0, \frac{1}{5}, \frac{1}{5}, \frac{3}{5}\right)$ & & $\downarrow j$ \\
\hline$\left(0, \frac{1}{5}, \frac{1}{2}, \frac{1}{5}, \frac{1}{10}\right)$ & & $\downarrow j$ \\
\hline$\left(0, \frac{1}{5}, \frac{3}{10}, \frac{2}{5}, \frac{1}{10}\right)$ & & $\downarrow j$ \\
\hline$\left(0, \frac{1}{5}, \frac{1}{5}, \frac{1}{2}, \frac{1}{10}\right)$ & & $\downarrow j$ \\
\hline$\left(0, \frac{1}{5}, \frac{1}{5}, \frac{2}{5}, \frac{1}{5}\right)$ & & $\downarrow j$ \\
\hline$\left(0, \frac{1}{5}, \frac{1}{5}, \frac{1}{5}, \frac{2}{5}\right)$ & & $\downarrow j$ \\
\hline
\end{tabular}


Table 2. Coherent systems with size 5

\begin{tabular}{lcc}
\hline $\mathbf{p}$ & $(n-j) h(j+1)$ & $j \tilde{r}(j)$ \\
\hline$\left(0, \frac{9}{10}, \frac{1}{10}, 0,0\right)$ & $\uparrow j$ & $\downarrow j$ \\
$\left(0, \frac{4}{5}, \frac{1}{5}, 0,0\right)$ & $\uparrow j$ & $\downarrow j$ \\
$\left(0, \frac{7}{10}, \frac{3}{10}, 0,0\right)$ & $\uparrow j$ & $\downarrow j$ \\
$\left(0, \frac{3}{5}, \frac{2}{5}, 0,0\right)$ & $\uparrow j$ & $\downarrow j$ \\
$\left(0, \frac{7}{10}, \frac{1}{5}, \frac{1}{10}, 0\right)$ & $\uparrow j$ & $\downarrow j$ \\
$\left(0, \frac{3}{5}, \frac{3}{10}, \frac{1}{10}, 0\right)$ & $\uparrow j$ & $\downarrow j$ \\
$\left(0, \frac{1}{2}, \frac{3}{10}, \frac{1}{5}, 0\right)$ & $\uparrow j$ & $\downarrow j$ \\
$\left(0, \frac{1}{2}, \frac{3}{10}, \frac{1}{5}, 0\right)$ & $\uparrow j$ & $\downarrow j$ \\
$\left(0,0, \frac{9}{10}, \frac{1}{10}, 0\right)$ & $\uparrow j$ & $\downarrow j$ \\
$\left(0,0, \frac{4}{5}, \frac{1}{5}, 0\right)$ & $\uparrow j$ & $\downarrow j$ \\
$\left(0,0, \frac{3}{5}, \frac{2}{5}, 0\right)$ & $\uparrow j$ & $\downarrow j$ \\
\hline
\end{tabular}

Ross (1980) proved that the number $N$ of failed components at the time a system fails has the discrete IFRA property. As elaborated in [Navarro and Samaniego (2017)], this fact implies that the signature of a system of arbitrary order $n$ cannot have internal zeros, that is, there exist no integers $i \in\{1, \ldots, n-2\}$ and $j \in\{2, \ldots, n-i\}$ for which $p_{i}>0$ and $p_{i+j}>0$ while $p_{i+1}=\cdots, p_{i+j-1}=0$. They also give an elementary proof of this result. Looking at Tables 1 and 2 , we may conjecture that every signature vector that can be written $\left(p_{1}, p_{2}, \ldots, p_{j}, 0,0, \ldots, 0\right)$ for some $j<n\left(p_{1}, p_{2}, \ldots, p_{j}, 0,0, \ldots, 0\right)$ for satisfies the condition (2.8) and a that every signature vector that can be written as $\left(0,0, \ldots, 0, p_{j}, p_{j+1}, \ldots, p_{n}\right)$ for some $j>1$ satisfies the condition (2.10) in Theorem 2.3.

In the following, we characterize all possible coherent systems of sizes 4 and 5 for which the results of Theorem 2.3 (c) can be applied.

Table 3. Coherent systems with sizes 4 and 5

\begin{tabular}{lcl}
\hline $\mathbf{p}$ & $\frac{u \Psi^{\prime}(u)}{\Psi(u)}>0$ & $\frac{(1-u) \Psi^{\prime}(u)}{\Psi(u)}<0$ \\
\hline$\left(0, \frac{1}{2}, \frac{1}{2}, 0\right)$ & $\downarrow u \in(0,0.667)$ & $\downarrow u \in(0.667,1)$ \\
$\left(0, \frac{2}{3}, \frac{1}{3}, 0\right)$ & $\downarrow u \in(0,0.708)$ & $\downarrow u \in(0.708,1)$ \\
$\left(0, \frac{1}{3}, \frac{2}{3}, 0\right)$ & $\downarrow u \in(0,0.61)$ & $\downarrow u \in(0.61,1)$ \\
& & \\
\hline $\mathbf{p}$ & $\frac{u \Psi^{\prime}(u)}{\Psi(u)}>0$ & $\frac{(1-u) \Psi^{\prime}(u)}{\Psi(u)}<0$ \\
\hline$\left(0, \frac{7}{10}, \frac{3}{10}, 0,0\right)$ & $\downarrow u \in(0,0.767)$ & $\downarrow u \in(0.767,1)$ \\
$\left(0, \frac{3}{5}, \frac{2}{5}, 0,0\right)$ & $\downarrow u \in(0,0.75)$ & $\downarrow u \in(0.75,1)$ \\
$\left(0, \frac{2}{5}, \frac{3}{5}, 0,0\right)$ & $\downarrow u \in(0,0.702)$ & $\downarrow u \in(0.702,1)$ \\
$\left(0, \frac{4}{5}, \frac{1}{5}, 0,0\right)$ & $\downarrow u \in(0,0.461)$ & $\downarrow u \in(0.461,1)$ \\
$\left(0,0, \frac{3}{5}, \frac{2}{5}, 0\right)$ & $\downarrow u \in(0,0.546)$ & $\downarrow u \in(0.546,1)$ \\
\hline
\end{tabular}

Again from Table 3, we conjecture that for a system with signature vector of the type 
$\left(0,0,0, \ldots, p_{j}, \ldots, p_{k}, 0,0, \ldots, 0\right)$, for some $2<j<k<n-1$, the conditions for the likelihood ratio order in Theorem 2.3 are satisfied.

Navarro et al. (2013) also obtained some stochastic comparisons results for coherent systems with dependent but identically distributed components. Let $T_{X}$ and $T_{Y}$ be the lifetimes of two coherent systems with the same structure and with iid component lifetimes and common absolutely continuous cumulative distribution functions F and G, respectively. Let $h$ be the common domination function and let us assume that it is twice differentiable. Among other results, Navarro et al. (2013) proved the following result in Theorem 2.6 (iv):

$$
\text { If } X \geq_{\ell r} Y \text { and } \frac{u h^{\prime \prime}(u)}{h^{\prime}(u)} \text { is nonnegative and decreasing in }(0,1) \text {, then } T_{X} \geq_{\ell r} T_{Y}
$$

We now provide a counterexample to illustrate that the condition (iv) in Theorem 2.6 of Navarro et al. (2013) for establishing likelihood ratio ordering is not satisfied, but it satisfies the conditions of our main result on likelihood ratio ordering between systems.

Example 3.5. Consider a coherent system of order 4 with signature vector $\mathbf{p}=\left(0, \frac{2}{3}, \frac{1}{3}, 0\right)$, and lifetime $T=\max \left(\min \left(X_{1}, X_{2}\right), \min \left(X_{3}, X_{4}\right)\right)$, where $X_{1}, X_{2}, X_{3}, X_{4}$ are independent and identically distributed. As shown in Table 1 of Navarro et al. (2013) the domination function of this system is

$$
h(u)=2 u^{2}-u^{4}
$$

and

$$
\Delta_{5}(u)=\frac{u h^{\prime \prime}(u)}{h^{\prime}(u)}=\frac{4 u-12 u^{3}}{4\left(u-u^{3}\right)} .
$$

The graph of the function $\Delta_{5}(u)$ is given in Figures 6. It is seen that $\Delta_{5}(u)$ is not always nonnegative. Therefore the condition (iv) in Theorem 2.6 of Navarro et al. (2013) for establishing likelihood ratio ordering is not satisfied, but the conditions $(i)$ and $(i i)$ in Theorem $2.3(c)$ are satisfied as seen from Table 3. Hence $\left.X \geq_{l r} Y \Longrightarrow T_{\mathbf{X}} \geq_{l r} T_{\mathbf{Y}}\right)$. These observations reveal that the conditions established in Theorem 2.3 are quite general.

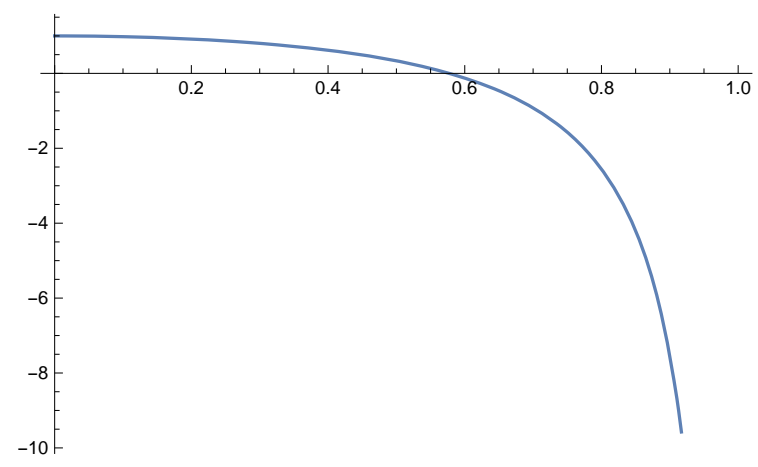

Figure 6: Plot of $\Delta_{5}(u)$, for all $u \in(0,1)$ 


\section{Conclusions}

In this paper, we consider the problem of stochastically comparing the lifetimes $T_{\mathbf{X}}(\mathbf{p})$ and $T_{\mathbf{Y}}(\mathbf{q})$ of two coherent systems with signature vectors $\mathbf{p}$ and $\mathbf{q}$ of the same size and with iid component lifetimes distributed according to $X$ and $Y$, respectively. The results established in this paper generalize some of the known results in the literature. Most of the existing results in the literature deal with the case when the two coherent systems have component lifetimes which are identically distributed. In this paper, we first consider the case when a coherent system operates under two different sets of independent and identically distributed component lifetimes. We find simple sufficient conditions on the distribution of the signature vector under which the two systems are stochastically ordered. In particular, We show that if $(n-j) h_{\mathbf{p}}(j+1)$ $\left(j \tilde{h_{\mathbf{p}}}(j)\right)$ is increasing in $j$ (decreasing in $j$ ) for any $j \geq 1$ and $X_{1} \geq_{h r(r h)} Y_{1}$, then a system with lifetime $T_{\mathbf{X}}(\mathbf{p})$ is larger than a system with lifetime $T_{\mathbf{Y}}(\mathbf{p})$ according to the hazard rate order (the reversed hazard rate order). We also give sufficient conditions on the signature vectors for a similar result for likelihood ratio order. Then we combine these new results with (1.4) to compare two coherent systems consisting of components with different lifetime distributions and also with possible different signatures. We characterize possible coherent systems of size 3,4 and 5 for which the above results can be applied; and also the proposed two conjectures for coherent systems of size $n>5$ are true. It will be interesting to examine whether our conjectures are true. We plan to pursue this problem in the near future.

\section{Acknowledgments}

The authors are grateful to the Editor and two anonymous referees for their valuable comments and suggestions on an earlier version of this manuscript which resulted in the present improved version. The simpler proof of Lemma 2.1 is due to one of the referees. Our original proof was based on theory of total positivity. The research of Baha-Eldin Khaledi was partially supported by Ordered and Spatial Data Center of Excellence of Ferdowsi University of Mashhad, Iran.

\section{References}

[Belzunce et al.(2001a)] Belzunce, F., Franco, M., Ruiz, J.M., and Ruiz, M.C., (2001). On partial orderings between coherent systems with different structure. Probability in the Engineering and Informational Sciences, 15, 273-293.

[Gupta (2002, p. 839)] Gupta, R.C. (2002). Reliability of a $k$ out of $n$ System of Components Sharing a Common Environment. Applied Mathematics Letters, 15, 837-844.

[Karlin(1968, p. 17)] Karlin, Samuel. (1968). Total Positivity. Stanford, California: Stanford University Press. 
[Khaledi and Shaked (2007)] Khaledi, B., and Shaked, M. (2007). Ordering conditional lifetimes of coherent systems. Journal of Statistical Planning and Inference, 137, 1173-1184.

[Kochar et al. (1999)] Kochar, S., Mukerjee, H., and Samaniego, F. (1999). The Signature of a coherent system and its application to comparisons among systems. Naval Research Logistics, 46, 507-523.

[Müller and Stoyan (2002)] Müller, A., and Stoyan, D. (2002). Comparison Methods for Stochastic Models and Risks. New York: John Wiley \& Sons.

[Navarro et al. (2008)] Navarro, J., Samaniego, F.J., Balakrishnan, N. and Bhattacharya, D.(2008). Applications and extensions of system signatures in engineering reliability. Naval Reserach Logististic,55,313327.

[1] [Navarro2010] Navarro J, Samaniego FJ, Balakrishnan N. The joint signature of coherent systems with shared components. Journal of Applied Probability 2010; 47:235253.

[Navarro and Samaniego (2017)] Navarro, J. and Samaniego, F. J. (2017). An elementary proof of the "no internal zeros" property of system signatures. Mathematical Methods in Reliability (MMR) Conference held in Grenoble, France in $201 \%$.

[Nanda et al. (1998)] Nanda, A.K., Jain, K., and Singh, H. (1998). Preservation of some partial orderings under the formation of coherent systems. Statistics and Probability Letters, 39, 123-131.

[Ross et al. (1980)] Ross, S.M., Shahsahani, M. and Weiss, G. (1980). On the Number of Component Failures in Systems Whose Components Are Exchangeable. Mathematics of Operrations Rsearch, 5, 358-365.

[Samaniego (1985)] Samaniego, F. J. (1985). On closure of the IFR class under formation of coherent systems. IEEE Transactions on Reliability, 34, 69-72.

[Samaniego (2007)] Samaniego, F. J. (2007). System Signatures and Their Applications in Engineering Reliability. New York: Springer Verlag.

[Shaked and Shanthikumar (2007)] Shaked, M., and Shanthikumar, J. G. (2007). Stochastic Orders. New York: Springer Verlag.

[Zhang (2010)] Zhang, Z. (2010). Ordering conditional general coherent systems with exchangeable components. Journal of Statistical Planning and Inference, 140, 454-460.

[Zhang and Meeker (2013)] Zhang, Z., and Q. Meeker, W. (2013). Mixture Representations of Reliability in Coherent Systems and Preservation Results under Double Monitoring. Communications in Statistics-Theory and Methods, 42, 385-397. 